\title{
Diseño del álabe de un aerogenerador horizontal de baja potencia
}

\section{Design of the blade of a low-power horizontal axis wind turbine}

\section{Cristhian Leonardo Pabón Rojas ${ }^{{ }^{*}}$, PhD. Elkin Flórez S ${ }^{2 *}$, PhD. Juan Carlos Serrano Rico ${ }^{3 *}$}

\author{
${ }^{1}$ Ingeniero Mecánico. cristt996@gmail.com \\ ${ }^{2}$ Doctor en Ingeniería Mecánica, Fluidos y Aeronáutica. eflorez@unipamplona.edu.co \\ ${ }^{3}$ Doctor en Ingeniería Mecánica. jcserrano@unipamplona.edu.co \\ *Programa Ingeniería Mecánica. Universidad de Pamplona
}

\section{Resumen}

Actualmente la energía eólica ha tenido gran auge dentro de las energías renovables; las turbinas que se emplean y sus álabes han sido modificadas para aumentar la eficiencia a la hora de extraer energía del viento. En este documento, se realiza el diseño de un álabe para un aerogenerador de baja potencia y baja velocidad, implementando distintos perfiles aerodinámicos. Estos perfiles fueron escogidos según su rendimiento aerodinámico el cual fue determinado por medio del software XFOIL. Basado en la teoría de momentum de elemento de alabe BEM por sus siglas en inglés, se realiza un rediseño continuo hasta que fuese capaz de extraer la máxima cantidad de energía a partir de la maximización de la relación sustentación/arrastre. Prestando atención a los problemas de desprendimiento de la capa límite y retardando la separación, que se presentan en los aerogeneradores expuestos a bajas velocidades de viento. Los perfiles aerodinámicos que fueron seleccionados para el diseño son: WORTMANN FX60-126 empleado en la raíz, el perfil SG6043 para la sección media por su gran comportamiento en el rango de número de Reynolds de diseño. Y finalmente el perfil SG6042 para la punta donde se encuentran los menores números de Reynolds. El diseño tuvo en cuenta valores de Reynolds entre 59000 y 32000. Obteniendo analíticamente 96,82W con un diámetro de $1,5 \mathrm{~m}$.

Palabras clave: energía eólica, álabe, perfil aerodinámico.

\section{Abstract}

Currently, wind energy has taken great increase in renewable energies; the turbines that are used and their blades have been modified to increase the efficiency when extracting energy from the wind. In this paper, the design of a blade for a low power and low wind turbine is made, implementing different airfoils. These airfoils were chosen according to their aerodynamic performance which was determined in the XFOIL software. Based on the BEM blade element momentum theory, a continuous redesign is made until it is able to extract the maximum energy from the lift/drag rate bigger. Paying attention to the problems of limit layer separation and retarding separation, which occur in wind turbines exposed to low wind speeds. The airfoils that were selected for the design are: WORTMANN FX60-126 used in the root, SG6043 airfoil for the middle section for its great performance in the Reynolds number range of design and finally the SG6042 airfoil for the tip where the lowest Reynolds numbers are found. This design considered Reynolds values between 59,000 and 32,000. Obtaining analytically 96,82W with a 1,5m diameter.El resumen en inglés

Keywords: wind energy, blade, airfoil, aerodynamics 


\section{Introducción}

La creciente preocupación y toma de conciencia en torno a minimizar los efectos nocivos sobre el medio ambiente, así como el uso eficiente de la energía, el alza del precio del petróleo en los años setenta, junto con el mal aprovechamiento de los recursos naturales y el agotamiento de los recursos que no son renovables promueven la búsqueda de fuentes alternativas de generación de energía. Una de estas fuentes es la energía eólica, esta situación deriva en un mayor aprovechamiento del recurso eólico, impulsado por su bajo impacto en el medio ambiente, una imagen favorable, y ahora es visto como una opción importante en el mercado energético. (Moragues \& Rapallini, 2003). Hoy en día la energía eólica es la de mejor relación costo beneficio, hablando de energías renovables. La necesidad de energías alternativas otorga un empuje a la utilización de tecnologías de energía renovables como esta. (Jamieson, 2018).

La extracción de la energía proveniente del viento tiene un futuro prometedor, la energía eólica será una de las principales fuentes de energía eléctrica. En Europa se espera que para el año 2050 entre un 31 y un $48 \%$ de la energía producida, sea de origen eólico. A pesar de que la creación de centrales de grandes potencias ha disminuido por las crisis financieras que se han visto, el interés en la energía eólica no se ha perdido, en especial el interés sobre las micro turbinas. Turbinas de pequeño tamaño que pueden ser usadas de manera urbana y generar energía de escala doméstica. Se hace interesante el comportamiento de estas turbinas con la variabilidad que tiene el viento en los terrenos urbanos. (Scappatici, y otros, 2016). A pesar de lo dicho anteriormente hablando del contexto nacional, Colombia es un país que en su historia ha dependido por mucho tiempo de la energía proveniente de fuentes fósiles. Con un consumo de estas, del $78 \%$ contra un $22 \%$ de energía renovable. Pero la crisis petrolera y reducción de otras fuentes fósiles alarma al mundo y a Colombia, un país fuerte en recursos como el petróleo, gas y carbón con problemas para suministrar energía a todo el país por el aislamiento topográfico y la variación climática haciendo importante las fuentes no convencionales para la generación de energía, donde la incertidumbre de precios en el combustible y la propia sostenibilidad de los recursos hacen presión para la implementación de lo no convencional. (Soto Gutierrez, 2016).

Pero la potencia contenida en el viento no es igual a la captada por un aerogenerador, el alemán Albert Betz en 1920 calculó la máxima eficiencia que puede tener una turbina eólica. Ese valor es 16/27 (0,593). Desde entonces es conocido como el límite de Betz. Límite que fue deducido también por el británico Lanchester cerca de la misma época y el científico ruso Joukowsky y hasta el día de hoy es un límite que no es posible superar con una turbina eólica. (Jiang, Li, \& Cheng, 2015).

La generación de energía eléctrica a partir de la velocidad del viento, básicamente requiere un rotor conectado a un generador eléctrico. Por su trabajo, el diseño aerodinámico del rotor cumple un papel muy importante para la maximización del aprovechamiento de la energía cinética. En particular, el rotor está compuesto por el cubo y los álabes, estas últimas son consideradas partes de la mayor importancia para el aerogenerador. (Mulugeta \& Gerawork, 2017). La eficiencia de una turbina eólica depende del diseño de varios elementos del álabe: la forma del perfil aerodinámico, el tamaño de la cuerda, el ángulo de ataque o de giro del perfil o la velocidad de giro. Son elementos que deben ser diseñados tomando en cuenta las condiciones de viento. Hay que prestar atención en especial al diseño del perfil aerodinámico y el ángulo de ataque, críticos para la capacidad de producir potencia por parte del rotor. La relación entre la sustentación y arrastre para cada perfil, tiene su propio ángulo de 
ataque optimo donde encuentra su mejor rendimiento. (Carlin, Laxson, \& Muljadi, 2003). El funcionamiento de las turbinas en pequeño tamaño y en zonas urbanas, presenta obstáculos como los problemas aerodinámicos del bajo número de Reynolds, considerado bajo por debajo de 500.000. El número de Reynolds se relaciona de manera directa con el pequeño tamaño de los álabes, y la baja velocidad del viento en el que funcionan estas micro-turbinas. Los problemas que se pueden presentar son: la separación de la capa laminar y el aumento del arrastre en los álabes por la creación de una burbuja de separación, eventos que disminuyen la eficiencia. (Singh, Ahmed, Zullah, \& Lee, 2012).

Para el diseño de los alabes del rotor, se recaba información sobre perfiles aerodinámicos con funcionamiento para bajos valores del número de Reynolds y se usan softwares que hagan posible la evaluación del desempeño aerodinámico de los perfiles. Un ejemplo de herramienta utilizada para este fin, es el software XFOIL, con una buena versatilidad para evaluar el comportamiento de perfiles a diferentes números de Reynolds y la funcionalidad al predecir distribuciones de presión y coeficientes de sustentación y coeficientes de arrastre del perfil entre otras características. (Drela, 1989) Con la información obtenida sobre el rendimiento aerodinámico de los perfiles, se puede aplicar teorías de diseño, como es la teoría BEM (Blade Element Momentum theory, por sus siglas en inglés) se basa en la teoría de cantidad de movimiento referida al análisis de un volumen de control, y la cantidad de energía que se puede obtener de él, combinándola con la teoría de elemento de álabe que analiza las fuerzas en una sección del aspa en función de su geometría, (Manwell, Mcgowan, \& Rogers, 2009). Para lo que se espera, resulte en un diseño con buen rendimiento en la extracción de energía o lo que es lo mismo, un valor alto para el coeficiente de potencia. La utilización de teoría BEM como método de diseño para mejorar el rendimiento de las turbinas de pequeño tamaño es
221

una estrategia que se hace cada vez más común. La optimización del rotor es económicamente más razonable comparado con la implementación de aspas que varíen su ángulo de manera mecánica, ya que el control de la dirección tendría un costo muy alto en comparación del incremento de energía extraída. (Scappatici, y otros, 2016).

\section{Procedimiento y Resultados}

Como punto de partida para el diseño de aerogenerador se tienen en cuenta parámetros como el tamaño del aerogenerador (diámetro), la velocidad del viento de diseño y el número de secciones de perfiles aerodinámicos que serían utilizados en el diseño.

El desarrollo comienza con la recopilación de la información pertinente tanto sobre el método de diseño como sobre los perfiles aerodinámicos que se podían usar en una turbina eólica de pequeño tamaño.

El aspecto tenido en cuenta para la recopilación de perfiles fue el comportamiento para números de Reynolds por debajo de 500.000 y la utilización con anterioridad en estudios o trabajos relacionados con aerogeneradores de pequeño tamaño. Algunos de los perfiles están diseñados en familias y son recomendados para distintas zonas o secciones en el álabe para su mejor aprovechamiento. Es el caso de los perfiles S833, S834 y S835 diseñados cómo una familia de perfiles para aerogeneradores de eje horizontal para bajos valores de Reynolds. (Somers, 2005). También la utilización de la familia de perfiles SG6040, SG6042 Y SG6043 son conveniente al provenir de un grupo diseñado para aerogeneradores de eje horizontal con funcionamiento a baja velocidad. Con los valores de coeficiente de sustentación determinado para cierto número de Reynolds, y al compararlos con otros perfiles, teniendo como criterio de comparación, la mayor relación entre el coeficiente de sustentación y el coeficiente de arrastre para determinado número de Reynolds, 
222

pero con la dificultad de una variación en cuanto al ángulo de ataque del viento y el número de Reynolds. (Giguere \& Selig, 1998).

Estas familias se forman para disponer de un perfil con mejor rendimiento aerodinámico $\mathrm{y}$ estructural en las distintas secciones que tiene el álabe. Un perfil con espesor más grande tendrá una menor relación de sustentación, pero presentará una oportunidad de ser usado en la base del álabe, llamada raíz, para mejorar el desempeño estructural. Pero no solo fueron seleccionadas estas familias, también está el perfil aerodinámico SD7032 seleccionado por su utilización cómo perfil de un rotor de funcionamiento a bajos valores de Reynolds y por su rendimiento (Bayati, Belloli, \& Bernini, 2017). Otros perfiles que también han sido utilizados en aerogeneradores, son WORTMANN y FX63137, los cuales fueron usados para simulación de los álabes de un rotor de un pequeño aerogenerador, el cual fue diseñado usando teoría de diseño BEM y el código de Qblade. (Holst, Pechlivanoglou, Kohlrausch, \& Nayeri, 2016). Por último, está el perfil Clark Y (Basavaraj,
2016) junto con los perfiles FX60-126 como variación del FX63-127 para medir el comportamiento de nuevos diseños de aerogeneradores. Con base en esta revisión de perfiles aerodinámicos utilizados en aerogeneradores de baja potencia y baja velocidad, se logró una base de datos la cual se muestra en la Figura. 1 , la cual será el punto de partida para seleccionar los 3 perfiles con mejor desempeño aerodinámico que se utilizarían en el diseño del álabe del aerogenerador. Para esta clasificación se evaluó el comportamiento de los perfiles aerodinámicos, mediante el uso del software XFOIL y una versión más moderna del mismo software llamado XFLR5 con el mismo código de programación base que el XFOIL, pero con un entorno de trabajo mucho más amigable con el usuario. En la Figura 2 y Figura. 3 se ve un ejemplo del comportamiento de los perfiles seleccionados. En ellas se puede observar la relación ente el coeficiente de sustentación y el coeficiente de arrastre para diferentes números de Reynolds para diferentes ángulos de ataque. 
S833

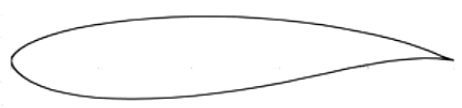

S834

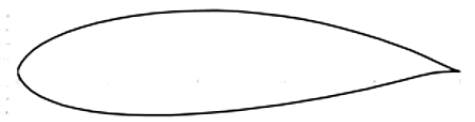

S835

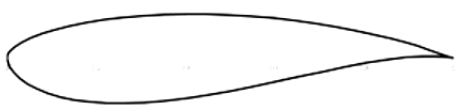

CLARK Y

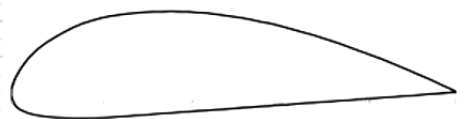

MID321A

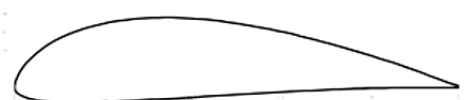

MID321B

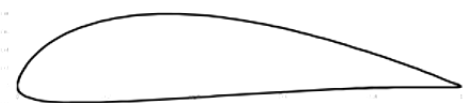

SG6040

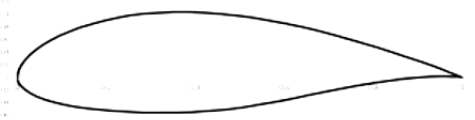

SG6042

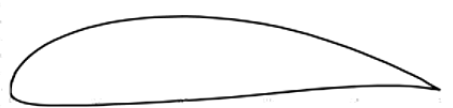

SG6043

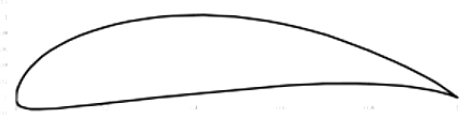

SD7032

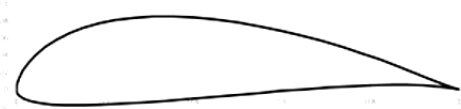

WORTMANN FX63-137

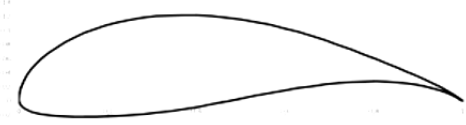

WORTMANN FX60-126

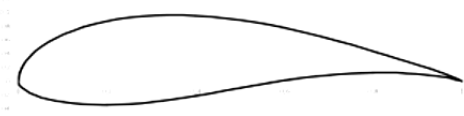

Figura. 1. Perfiles aerodinámicos de la base de datos.

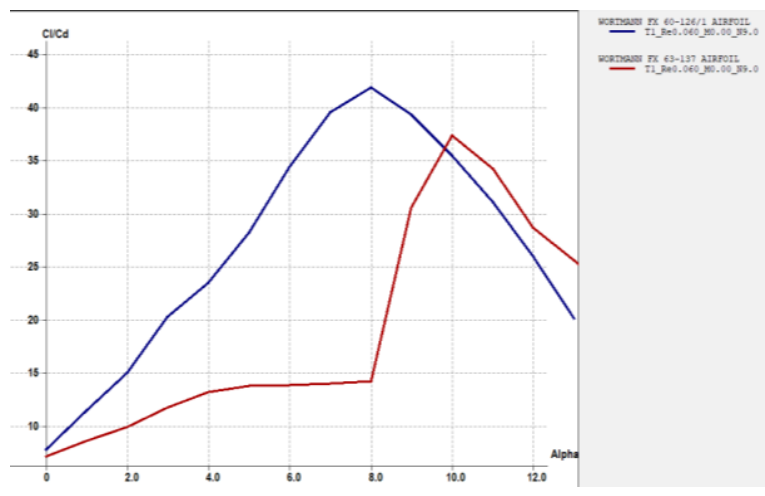

Figura. 2. Comportamiento $C_{L} / C_{D}$ vs Alpha para un número de Reynolds de 60.000 de los perfiles Wortmann FX60-126 y FX63-137,

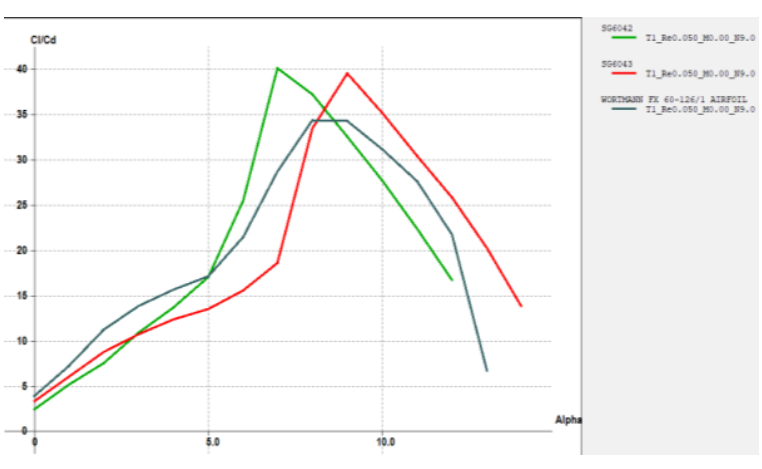

Figura. 3. Comportamiento $C_{L} / C_{D}$ vs Alpha para un número de Reynolds de 50.000 de los perfiles SG6042, SG6043 y Wortmann FX60-126.

\subsection{Selección de los perfiles}

En un rango para el número de Reynolds entre 50.000 y 150.000 se evaluaron parámetros como: la relación del coeficiente de sustentación y el coeficiente de arrastre vs el ángulo de ataque, el coeficiente de sustentación vs el ángulo de ataque, el coeficiente de sustentación vs el arrastre, además de tener en cuenta datos geométricos 
224

como el espesor máximo de los perfiles para su ubicación dentro del diseño del álabe. La relación entre la sustentación y el arrastre, es el parámetro importante en el diseño de los álabes para una turbina eólica. Para el diseño, se toma el valor máximo de esta relación. Este parámetro de comportamiento está ligado al número de Reynolds. Entre mayor sea el número de Reynolds mayor va a ser la sustentación obtenida por el perfil, mientras que, si el valor del número decrece, aumenta el arrastre mínimo en el perfil a la vez que disminuye la sustentación máxima. (Miley, 1982).

Los perfiles seleccionados preliminar mente fueron el Wortmann FX63-137 para la zona de la raíz del álabe, el perfil SG6043 para la zona media y para la zona más a la punta el SG6042. Pero luego se decide cambiar el perfil Wortmann FX63-137 por el perfil Wortmann FX60-126 demostrando un mejor comportamiento para los valores del número de Reynolds evaluados para la sección del álabe. Figura. 2.

\subsection{Calculo de la geometría del álabe}

La utilización del perfil Wortmann FX60-126 para la raíz es tomada por su mayor espesor. El perfil SG6043 es elegido para la zona media por su mejor comportamiento en todo el rango del número de Reynolds elegido. $\mathrm{Y}$ por último el perfil SG6042 es elegido para la punta por su buen comportamiento en los números de Reynolds más bajos y menor curvatura de perfil comparada con los otros seleccionados. Habiendo seleccionado los perfiles más adecuados, se procede a calcular la geometría con la que contarán a lo largo del álabe. es importante aclarar que el diseño hecho en este trabajo no tiene grandes análisis de su resistencia física ni de sus materiales en caso de una posible construcción. Este trabajo solo se basa en el diseño aerodinámico y deja aparte toda recomendación estructural o de fabricación. La única medida tomada en cuenta como se dijo anteriormente, es la distribución de los perfiles con el máximo espesor hacia la zona más cercana a la raíz del aspa.

Para el cálculo de la geometría se utilizó la teoría BEM para obtener el tamaño de la cuerda (c) que puede ser descrita como porcentaje del radio (R) del álabe, en este diseño ese radio de aerogenerador $\mathrm{R}$ es igual a $1,5 \mathrm{~m}$.

Para facilitar el diseño y la comprensión se dividió el álabe en 12 secciones que van en orden desde el centro de giro del rotor, hasta la sección 12 ubicada en la sección más alejada del eje de rotación, para cada una de estas secciones del álabe fue calculado el valor para la cuerda, esto es mostrado en la Figura. 4, también el ángulo de giro fue calculado en estas 12 secciones y su valor es descrito en grados para cada punto en la Figura.

5.

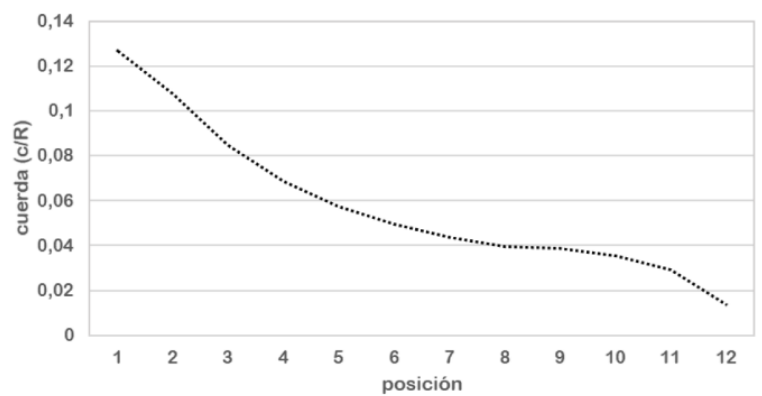

Figura. 4. cuerda del álabe.

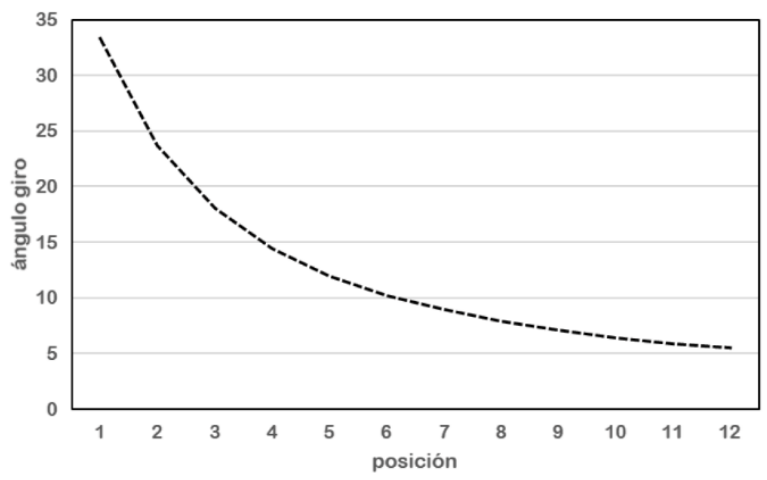

Figura. 5. ángulo de giro del álabe.

Luego de obtener los datos se lleva a cabo un análisis analítico mediante la misma teoría BEM expresada por Manwell, para el cálculo del coeficiente de potencia del diseño del álabe. 
225

Como resultado se obtiene un coeficiente de potencia analítico igual a $\mathrm{Cp}=0,4141$. Considerando el área de barrido por el álabe, resulta en una potencia de $96,82 \mathrm{~W}$, que al compararse con la máxima posible usando el límite de Betz igual a 138,55W. Corresponde a un 71,3\% del límite teórico de Betz y corresponde con otros trabajos como el de (Chirinos Garcia, Moreno Figueredo, \& Morales Salas, 2016) donde el coeficiente de potencia conseguido con el modelo matemático ronda el $37 \%$.

\subsection{CAD y simulación}

Con las medidas calculadas mediante el método BEM se realiza el CAD del álabe, con el fin de poder realizar una simulación y comparar aspectos que relacionen el comportamiento analítico con un modelo más acercado a la realidad como una simulación CFD.

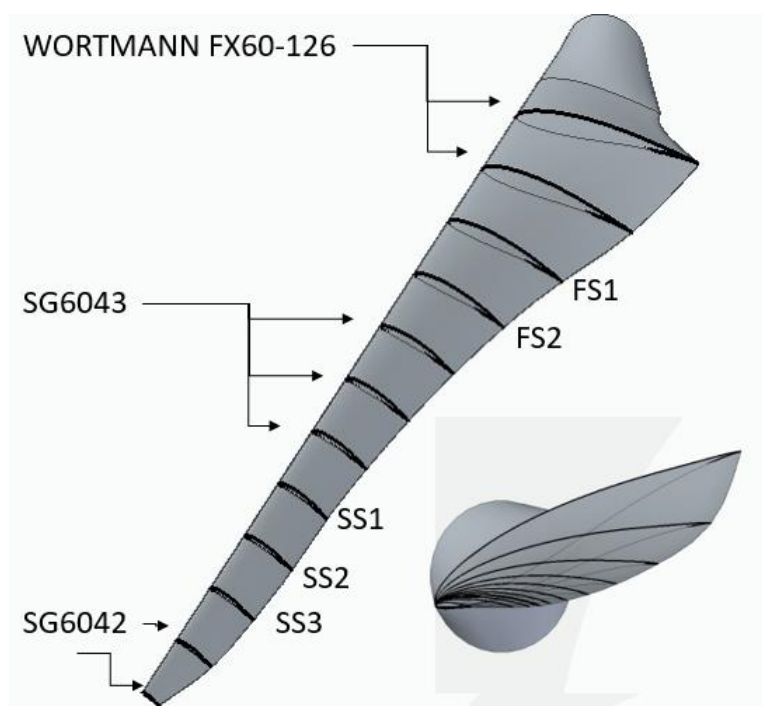

Figura. 6. CAD álabe, posición de los perfiles en las secciones.

El parámetro que se utilizó para la comparación, fue el coeficiente de presión para el valor del número de Reynolds que analíticamente interactuaba en cada sección del álabe. La comparación se realizó entre la gráfica obtenida en el software XFLR5 en cada sección y la obtenida en el software ANSYS. A continuación, se comparan las gráficas obtenidas mediante XFLR5 y ANSYS para la distribución del coeficiente de presión. La primera comparación se realiza a una distancia de $0,09 \mathrm{~m}$. en estas graficas el tamaño de la cuerda es tomada como una unidad. Así la cuerda es un valor adimensional que en el eje $\mathrm{X}$ al alcanzar el valor máximo de1 y significa que el coeficiente es evaluado a lo largo de la cuerda en la sección estudiada.
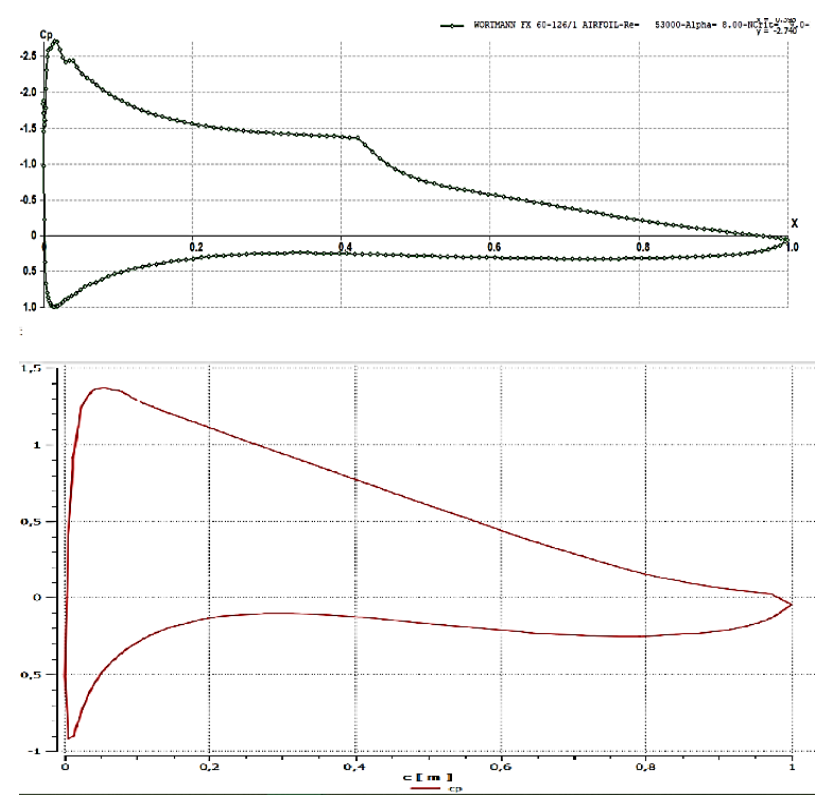

Figura. 7. Coeficiente de presión en el perfil de una sección del álabe que se encuentra a $0,9 \mathrm{~m}$ en dirección radial del eje de rotación usando XFLR5(grafica verde) y ANSYS (grafica roja).

Se observa gran similitud entre las gráficas en la Figura. 7 donde se aprecian, área y valores similares entre ambas herramientas computacionales, aunque para el valor de succión máxima en el extradós el valor obtenido con ANSYS es bastante menor, en el software XFLR5 se ve una caída de succión en el centro del perfil que no es visto en la gráfica del coeficiente de presión en ANSYS. A esta misma distancia radial 
226

analizando el campo de velocidades como es mostrado en la Figura. 8 se puede ver lo que sería una transición entre un flujo con alta velocidad hacia una disminución de esta velocidad en la sección media, la misma zona donde la energía cinética del flujo turbulento de la Figura. 9 aumenta hasta llegar a su máximo. Esta zona mencionada coincide con la distancia a la cual hay un cambio en el $C p$ del extradós en la Figura. 7. Adicional, en la Figura. 8 se puede ver el punto de "remanso", el punto donde la presión es máxima en el intradós y la velocidad del fluido es mínima. (Colosqui, Delnero, Marañón Di Leo, Colman, \& Boldes).

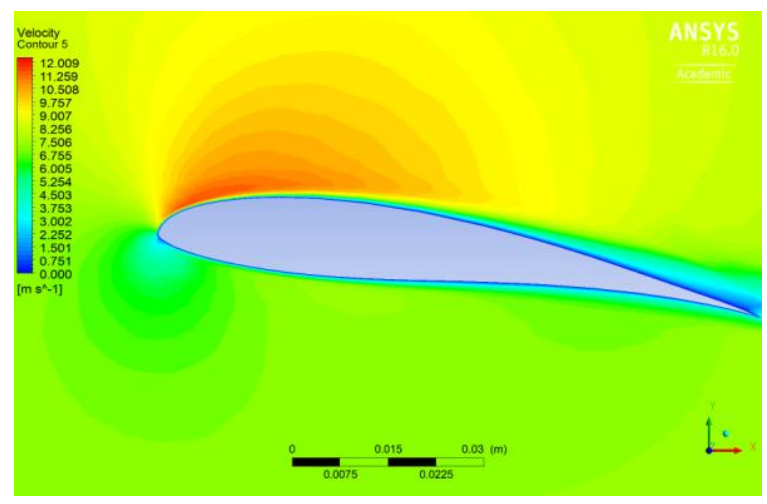

Figura. 8. Campo de velocidades para el perfil del álabe.

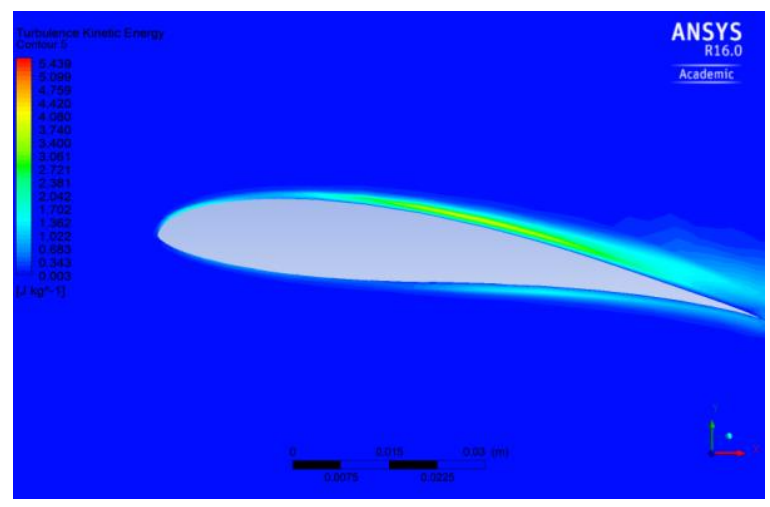

Figura. 9. Campo de energía cinética en el flujo turbulento para el perfil del álabe.

En la zona media del álabe se observa un mejor comportamiento en la simulación con ANSYS que con el software XFLR5. se observa una gran diferencia de presiones entre el intradós y el extradós. Y ambas graficas mantienen un comportamiento similar a lo largo del álabe. Como se observa en la Figura. 10.

Por otro lado, evaluando una zona alejada en sentido radial, desde el eje de rotación. a un radio de $0,69 \mathrm{~m}$, donde se encuentra un perfil SG6042. Se ve gran diferencia en los valores para el coeficiente de presión, los cuales son presentados en la Figura. 11.

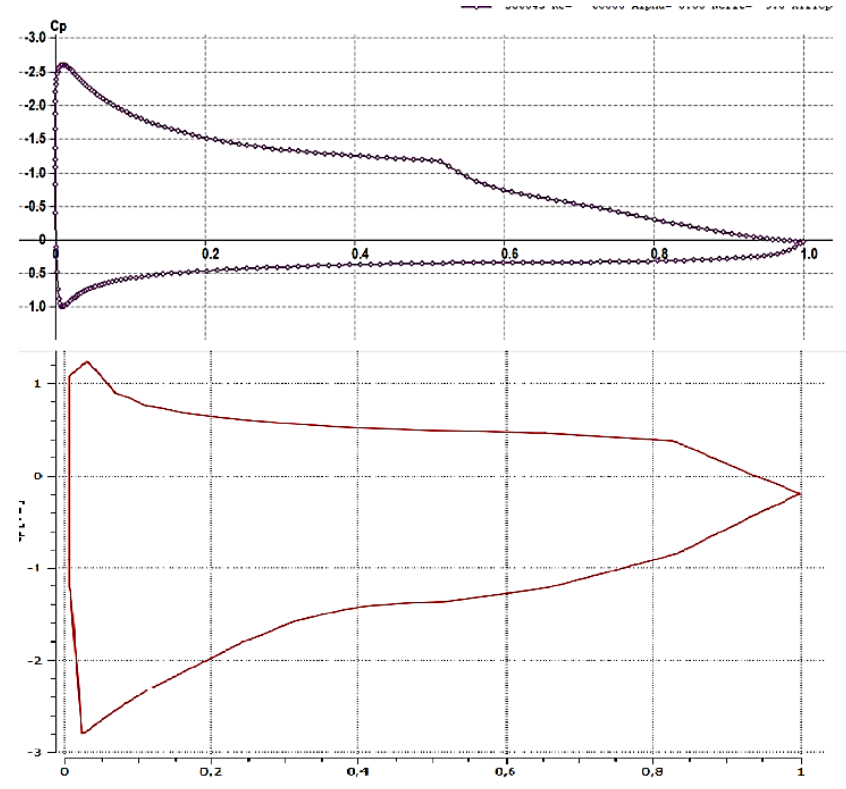

Figura. 10. Grafica coeficiente de presión para un radio $0,39 \mathrm{~m}$ en software XFLR5 (color morado) y software ANSYS (color rojo), hay que resaltar que en la gráfica de ANSYS los valores para el extradós se encuentran en la parte inferior. 

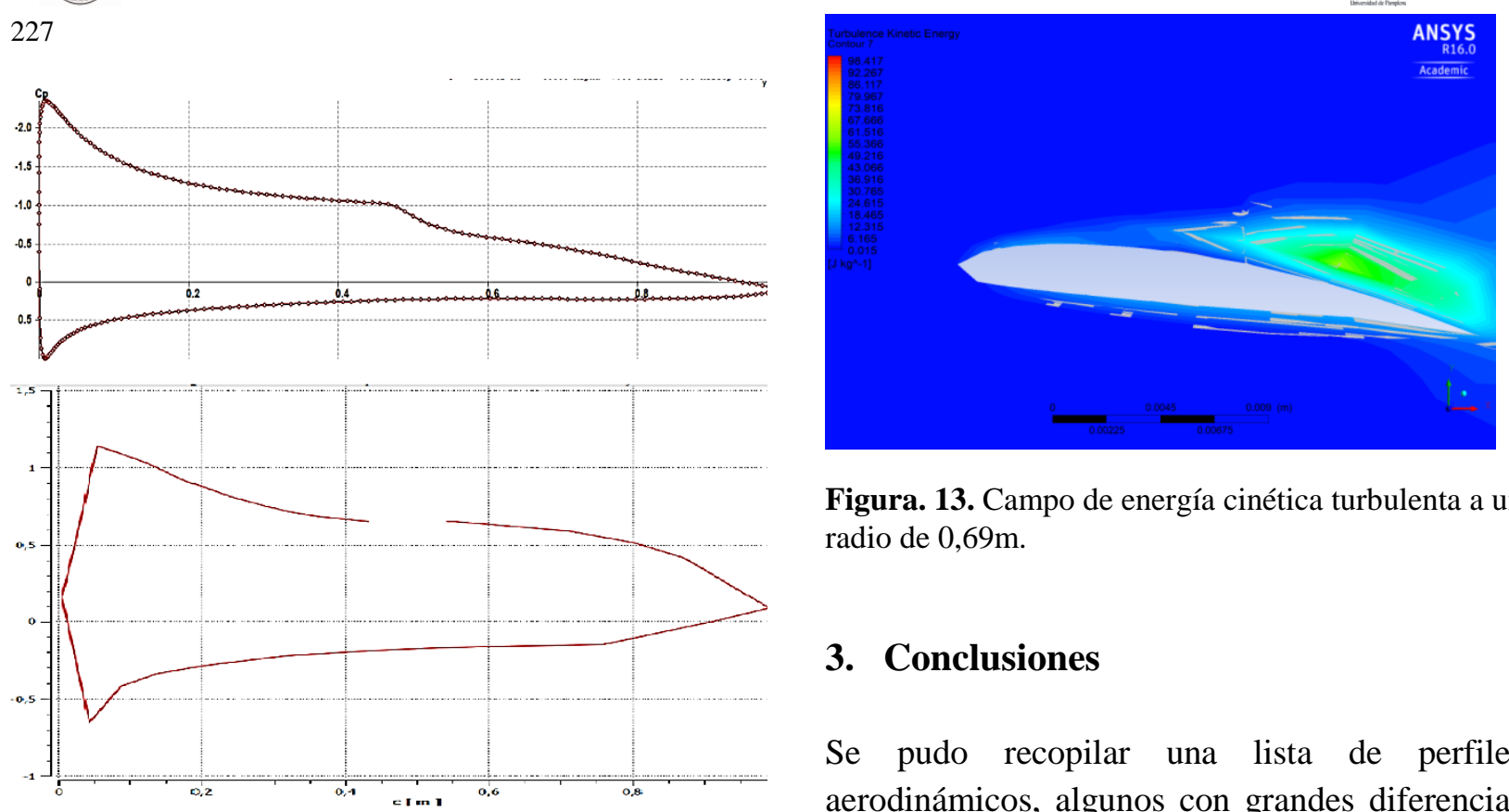

Figura. 13. Campo de energía cinética turbulenta a un radio de $0,69 \mathrm{~m}$.

\section{Conclusiones}

Se pudo recopilar una lista de perfiles aerodinámicos, algunos con grandes diferencias en su año de diseño y con un rendimiento aceptable para bajos valores de Reynolds. A pesar de ser unos más antiguos que otros, muchos mostraron tener un rendimiento prometedor. Gracias a este rendimiento, el uso de perfiles que no estaban dentro de la misma familia, se hace importante para los resultados finales. Aunque no se pudo utilizar algunos perfiles diseñados recientemente, por falta de información o permisos para su estudio, los perfiles usados mostraron un comportamiento de gran calidad para el trabajo, evidenciando por qué fueron objeto de estudio y constituyen una parte importante en el diseño de aerogeneradores.

La teoría utilizada para el diseño de los alabes, la teoría BEM, involucra una mezcla de variables tanto geométricas como analíticas que permiten un desarrollo en el diseño bastante completo. Permite un análisis detallado, que funciona para aspas de rotores que enfrenten vientos de baja velocidad según el objeto de este trabajo. La utilización de un álabe con diferentes perfiles muestra un prometedor rendimiento según los datos analíticos, con la capacidad de trabajar para valores muy bajos de número de Reynolds y una geometría reducida que permite utilizar menos material en caso de su fabricación, lo cual

Figura. 12. Campo de velocidad para perfil del álabe a un radio de $0,69 \mathrm{~m}$. 
228

minimiza los esfuerzos sobre los álabes. Resulta interesante la capacidad desarrollada para obtener energía del recurso eólico.

Analizando el comportamiento para la capacidad de extracción de potencia del álabe, se puede ver que al aumentar la velocidad nominal $\lambda$ aumenta el coeficiente de potencia, hasta acercarse a la punta donde la perdida aumenta de manera significativa. A pesar de eso el valor obtenido para el coeficiente de potencia es alto alcanzando un valor de $41,41 \%$.

Debe ser usada una herramienta de análisis CFD con mucha más profundidad, que permita la interpretación de resultados más relevantes, para una comparación con mayor facilidad.

Probablemente el modelo realizado no tenga las mejores características estructurales, esto por haber excluido un análisis en ese aspecto. Pero el enfoque en la parte aerodinámica abre la puerta a un punto de comparación para otros trabajos.

Entre más alejada es la distancia del centro de rotación, los valores para el coeficiente de presión obtenidos con ANSYS y XFLR5 varían más. A pesar de que el cálculo de la geometría se realizara teniendo en cuenta la perdida en la punta del diseño BEM. al XFLR5 solo tener cálculos basados en el número de Reynolds no tiene en cuenta panoramas que quizá ANSYS FLUENT sí, y es quizá el porqué de la diferencia tan grande en el comportamiento para uno y el otro.

\section{Referencias Bibliográficas}

Basavaraj, A. (2016). Computational Analysis of Airfoil Merging and its Effect on Performance of Lift Based Vertical Axis Wind Turbine. ASME 2016 International Mechanical Engineering Congress and Exposition.

Bayati, I., Belloli, M., \& Bernini, L. \&. (2017). Aerodynamic design methodology for wind tunnel tests of wind turbine rotors.
Journal of Wind Engineering and Industrial Aerodynamics, 217-227.

Carlin, P. W., Laxson, A. S., \& Muljadi, E. (2003). The history and state of the art of variable-speed wind turbine technology. Wind Energy, Wiley Online Library, 129-159.

Chirinos Garcia, J. J., Moreno Figueredo, C., \& Morales Salas, J. (2016). Modelo matemático para el diseño aerodinámico de los álabes de una turbina eólica de eje horizontal (TEEH). Ingeniería Energética, 37, 63-72.

Colosqui, C., Delnero, S., Marañón Di Leo, J., Colman, J., \& Boldes, U. (s.f.). CÁLCULO DE COEFICIENTES AERODINÁMICOS DE PERFILES DE BAJO REYNOLDS MEDIANTE EL METODO DE ELEMENTOS FINITOS.

Drela, M. (1989). XFOIL: An analysis and design system for low Reynolds number airfoils. Low Reynolds number aerodynamics, Springer, 1-12.

Giguere, P., \& Selig, M. S. (1998). New airfoils for small horizontal axis wind turbines. Journal of solar energy engineering, American Society of Mechanical Engineers, 108-114.

Holst, D., Pechlivanoglou, G., Kohlrausch, C., \& Nayeri, C. \&. (2016). sHAWT Design: Airfoil Aerodynamics Under the Influence of Roughness. ASME Turbo Expo 2016: Turbomachinery Technical Conference and Exposition.

Jamieson, P. (2018). Innovation in wind turbine design. John Wiley \& Sons.

Jiang, H., Li, Y., \& Cheng, Z. (2015). Performances of ideal wind turbine. Renewable Energy, 658-662.

Manwell, J., Mcgowan, J., \& Rogers, A. (2009). wind energy explained Theory, desing and aplication. Chichester: Jhon Wiley \& Sons Ltda. 
Miley, S. J. (1982). A catalog of low Reynolds number airfoil data for wind turbine applications.

Molina M, Tebaldi M, Bolognini N.2013.Eficiencia de difracción del registro de speckle modulados generados a partir de superficies reflecto-difusoras. Bistua:Revista de la Facultad de Ciencias Basicas. 10 (2):50-60.

Moragues, J., \& Rapallini, A. (2003). Energía eólica. Instituto Argentino de la Energ\ia "General Mosconi.

Mulugeta, B. A., \& Gerawork, A. (2017). Aerodynamic design of horizontal axis wind turbine blades. FME Transactions, 647-660.

Scappatici, L., Bartolini, N., Castellani, F., Astolfi, D., Garinei, A., \& Pennicchi, M. (2016). Optimizing the design of horizontal-axis small wind turbines: From the laboratory to market. Journal of Wind Engineering and Industrial Aerodynamics, 154, 58-68.

Singh, R., Ahmed, M., Zullah, M., \& Lee, Y.-H. (2012). Design of a low Reynolds number airfoil for small horizontal axis wind turbines. Renewable Energy, 6676.

Somers, D. M. ( 2005). S833, S834, and S835 Airfoils: November 2001--November 2002. National Renewable Energy Laboratory (NREL), Golden, CO., National Renewable Energy Laboratory (NREL), Golden, CO., .

Soto Gutierrez, J. J. (2016). Desarrollo de la energía eólica en Colombia. \{B.S.\} thesis, Fundación Universidad de América.
* Para citar este artículo: Pabón Rojas C.L; Flórez S E; Juan Carlos Serrano Rico J.C.Design of the blade of a low-power horizontal axis wind turbine.Revista Bistua.2019.17(1):219-229

+ Autor para el envió de correspondencia y la solicitud de las separatas: Flórez $\mathrm{S}$ E .Programa de Ingenieria Mecánica. Universidad de Pamplona, Pamplona, Colombia.eflorez@unipamplona.edu.co

Recibido: Marzo 23 de 2018

Aceptado: Agosto 20 de 2018 